\title{
FGF8 promotes cell proliferation and resistance to EGFR inhibitors via upregulation of EGFR in human hepatocellular carcinoma cells
}

\author{
YUANMIN PEI $^{1 *}$, XUELING SUN $^{1 *}$, XIWEI GUO ${ }^{1}$, HUASHAN YIN $^{1}$, LE WANG $^{2}$, FUGU TIAN $^{2}$, \\ HONGXI JING ${ }^{2}$, XIAOBO LIANG ${ }^{2}$, JUN XU $^{2}$ and PENGCHENG SHI ${ }^{2}$ \\ ${ }^{1}$ Department of General Surgery, Yidu Central Hospital of Weifang, Weifang Medical University, Weifang, Shandong 261000; \\ ${ }^{2}$ Shanxi Breast Cancer Center, Shanxi Cancer Hospital, Shanxi Medical University, Taiyuan, Shanxi 030001, P.R. China
}

Received November 18, 2016; Accepted June 13, 2017

DOI: $10.3892 /$ or.2017.5887

\begin{abstract}
Fibroblast growth factor 8 (FGF8), a member of the fibroblast growth factor (FGF) family, is upregulated in several human cancers, including HCC (HCC). Previous studies have demonstrated that FGF8 increased cell growth and invasion of tumor cells. In the present study we investigated whether FGF8 is involved in the cell proliferation and resistance to several drugs in human HCC cells. We stably overexpressed FGF8 by lentiviral transfection. In addition, we also added recombinant FGF8 instead of stably overexpressing FGF8 in human HCC cells. Stable overexpression of FGF8 or exogenous recombinant FGF8 resulted in significantly enhanced cell proliferation in human HCC cells. With the use of CellTiter-Glo assay for the determination of cell viability, we found that FGF8 increased the resistance to epidermal growth factor receptor (EGFR) inhibitors in human HCC cells. Additionally, the expression of EGFR was also upregulated by stably overexpressing FGF8 or exogenous recombinant FGF8. Yes-associated protein 1 (YAP1) was reported to upregulate the expression of EGFR. Moreover, we also found that FGF8 increased the expression of YAP1 and knockdown of YAP1 eliminated the upregulation of EGFR and the resistance to EGFR inhibition induced by FGF8. Our study provides evidence that FGF8 plays an important role in the resistance to EGFR inhibition of human HCC cells.
\end{abstract}

\section{Introduction}

Fibroblast growth factors (FGFs) are a large family of growth factors that signal through FGF receptors (FGFRs) to regulate

Correspondence to: Dr Pengcheng Shi, Shanxi Breast Cancer Center, Shanxi Cancer Hospital, Shanxi Medical University, Taiyuan, Shanxi 030001, P.R. China

E-mail: pengchshi@163.com

${ }^{*}$ Contributed equally

Key words: FGF8, HCC, resistance, YAP1, EGFR fundamental developmental pathways, controlling events such as cell proliferation, differentiation, cell survival, angiogenesis, and wound healing (1-3). Aberrant FGF signaling can promote tumor development by increasing tumor cell proliferation, invasion, survival and angiogenesis (3). Fibroblast growth factor 8 (FGF8) was first identified in the Shionogi mouse mammary carcinoma cell line SC-3 and its expression was induced by androgen $(4,5)$. FGF8 is rarely detected in normal adult tissues, but upregulated in several human cancers, including HCC (HCC) (6). It was reported that autocrine overexpression of FGF8 in phosphatase and tensin homolog (PTEN) deficiency of prostate epithelium could lead to prostate adenocarcinoma (7). Overexpression of FGF8 in prostate cancer is associated with decreased patient survival and persists in androgen-independent diseases (8).

The Hippo signaling pathway was initially defined as a regulator of proliferation, organ size, and cell differentiation $(9,10)$. Yes-associated protein 1 (YAP1) is a transcriptional coactivator in the Hippo signaling pathway $(11,12)$. Increased YAP1 expression or activity can promote the growth of tumor cells (13). It was reported that $\beta$-catenin-driven cancers require a YAP1 transcriptional complex for survival and tumorigenesis (14). Enforced YAP1 expression can enable tumor maintenance upon $\mathrm{Kras}^{\mathrm{G} 12 \mathrm{D}}$ extinction in a human pancreatic ductal adenocarcinoma model (15). Moreover, YAP1 amplification has been described as an essential oncogene in various types of human cancers, including human HCC (16).

Epidermal growth factor receptor (EGFR) signaling plays an important role in various cancers, including HCC (17). Overexpression of EGFR was also found in $\mathrm{HCC}$, and hence EGFR represents a valuable therapeutic target for the treatment of HCC $(18,19)$. EGFR antagonists were effective in human HCC cells (18). Gefitinib and erlotinib, two clinically approved EGFR chemical inhibitors, are used for the treatment of advanced HCC (20-25). However, some studies have demonstrated the resistance to EGFR inhibitors in human HCC cells (26-29).

The aim of the present study was to determine the effect of FGF8 in human HCC cells. Our data revealed that FGF8 promoted cell growth and the resistance to EGFR inhibition via upregulation of EGFR in human HCC cells. 


\section{Materials and methods}

Cell lines and culture. HepG2 and HCC-LM3 cell lines were purchased from the American Type Culture Collection (ATCC; Dallas, TX, USA). These cells were maintained in Dulbecco's modified Eagle's medium (DMEM) supplemented with 10\% fetal bovine serum (FBS), penicillin $(100 \mathrm{U} / \mathrm{ml})$ and streptomycin $(100 \mu \mathrm{g} / \mathrm{ml})$ in a humidified atmosphere of $5 \% \mathrm{CO}_{2}$ at $37^{\circ} \mathrm{C}$.

Reagents. 5-Fluorouracil (5-FU), doxorubicin, paclitaxel, oxaliplatin, erlotinib, gefitinib and RAD001 (Everolimus) were purchased from Selleck Chemicals LLC (Houston, TX, USA). Human recombinant FGF8 was purchased from PeproTech (Rocky Hill, NJ, USA).

Cell viability assay. Cells were seeded into white walled clearbottom 96-well plates and grown for $24 \mathrm{~h}$ before treatment with various concentrations of drugs. Cell viability was assessed after 24-h treatment by measuring the cellular ATP levels using Luminescent CellTiter-Glo assay (Promega, Madison, WI, USA). All drug treatments were performed at least in triplicate and the data was normalized to the vehicle-treated control and presented as the mean \pm SEM.

Lentivirus transduction. The lentivirus of the YAP1 shRNAs (PLKO.1 vector, target sequences: sh1-CAGGTGATACTATC AACCAAA; sh2-CAGGTGATACTATCAACCAAA) and FGF8 overexpressing ( $\mathrm{pCDH}$ vector) or their vector control lentivirus were obtained from Genesent (Shanghai, China). Viral infection was performed in 6-well plates using $2 \times 10^{6} \mathrm{HepG} 2$ or HCC-LM3 cells in a total volume of $2 \mathrm{ml}$ of lentiviral supernatant containing $8 \mu \mathrm{g} / \mathrm{ml}$ of Polybrene (Sigma-Aldrich, St. Louis, MO, USA). Two days after infection, $1 \mu \mathrm{g} / \mathrm{ml}$ of puromycin (Sigma-Aldrich) was added to the media and selected for one week. The expression levels of the target genes were determined by western blot analysis.

Real-time PCR. Gene expression levels were determined with real-time RT-PCR. Total RNA was isolated using TRIzol (Invitrogen, Carlsbad, CA, USA) and procedures were carried out as recommended by the manufacturer. Then, extracted RNA was subjected to reverse transcription using the PrimeScript II First Strand cDNA Synthesis kit (Takara, Dalian, China) according to the manufacturer's instructions. The primers for human VEGFR were: forward, 5'-AGGCACG AGTAACAAGCTCAC-3' and reverse, 5'-ATGAGGACATAA CCAGCCACC-3'. Real-time PCR was performed with a 7500 Fast Real-Time PCR system (Applied Biosystems, Foster City, CA, USA) using SYBR-Green Gene expression analysis (Tiangen, Shanghai, China). EGFR expression was normalized to GAPDH. Ct values of the target genes were subtracted by that of the internal control GAPDH. The relative expression levels of EGFR were assessed by the $2^{-\Delta \Delta \mathrm{Ct}}$ method.

Colony formation assays. HepG2 or HCC-LM3 human HCC cell lines were seeded into 6 -well plates with 500 cells/well in triplicate and cultured for $24 \mathrm{~h}$. Then the cells were treated with exogenous recombinant FGF8 or erlotinib and cultured for 14 days. The colonies were fixed with $10 \%$ formaldehyde, stained with crystal violet (0.4 g/l; Sigma, St. Louis, MO,
USA). The number of colonies was counted using grid. Three independent experiments were performed and the data are presented as the mean $\pm \mathrm{SD}$.

Tumor sphere formation assay. Single shNC or sh1-YAP1 of HepG2 cells were plated at a density of 1,000 cells $/ \mathrm{ml}$ onto a 6-well ultralow attachment plate (500 cells/well; Corning, Lowell,MA, USA). Cells were grown in serum-free DMEM/F12 medium supplemented with $4 \mu \mathrm{g} / \mathrm{ml}$ of insulin (Sigma-Aldrich), B27, $20 \mathrm{ng} / \mathrm{ml}$ of EGF, and $20 \mathrm{ng} / \mathrm{ml}$ of basic FGF (Invitrogen, Carlsbad, CA, USA). The cells were then incubated at $37^{\circ} \mathrm{C}$ in a humidified atmosphere containing $5 \% \mathrm{CO}_{2}$ for 14 days.

Western blot analysis. Cells were trypsinized and washed 3 times with phosphate-buffered saline (PBS) before being lysed on ice for 30 min with RIPA lysis buffer containing and complete protease/phosphatase inhibitor cocktail (Roche Life Science, Mannheim, Germany). The lysate was centrifuged at $13,000 \mathrm{rpm}$ for $20 \mathrm{~min}$ at $4^{\circ} \mathrm{C}$ and the supernatant was collected. Protein concentration was determined by Bradford protein assay. The proteins were separated by SDS-PAGE gel electrophoresis and then transferred onto polyvinylidene difluoride (PVDF) membranes (Millipore Inc., Billerica, MA, USA). The membranes were blocked with 5\% skimmed milk in TBST for $1 \mathrm{~h}$ and then were incubated with primary antibodies overnight at $4^{\circ} \mathrm{C}$. Primary antibodies: rabbit polyclonal to FGF8 $(1: 1,000$, ab81384), rabbit monoclonal (EP38Y) to EGFR $(1: 1,000$, ab52894), rabbit monoclonal (EP1674Y) to YAP1 $(1: 1,000$, ab52771), mouse monoclonal to $\beta$-actin $(1: 3,000$, ab8226) were all obtained from Abcam (Cambridge, MA, USA). After being washed with TBST 3 times, the membranes were probed with HRP-conjugated secondary antibodies in TBST for $1 \mathrm{~h}$ at room temperature, and then washed with PBST three times. The results were analyzed using a chemiluminescence method (ECL; Millipore Inc.).

Statistical analysis. Data are presented as the means \pm SD and analyzed using Student's t-test. P-values of $<0.05$ were considered to indicate a statistically significant result.

\section{Results}

FGF8 enhances the proliferation of human HCC cells. To determine the potential significance of FGF8 in the proliferation of human HCC cells, exogenous recombinant human FGF8 was added to HepG2 and HCC-LM3 human HCC cell lines. Colony formation assays were used to examine the proliferation of human HCC cells affected by exogenous human recombinant FGF8. As shown in Fig. 1A, colony formation assays revealed that exogenous recombinant FGF8 increased the proliferation of HepG2 and HCC-LM3 cells in vitro. To extend this analysis, we also generated stable HepG2 or HCC-LM3 cell lines that stably expressed human FGF8 (Fig. 1B). We found that stable overexpression of FGF8 also increased the proliferation of HepG2 or HCC-LM3 cells in vitro (Fig. 1B).

FGF8 promotes the resistance to EGFR inhibitors of human HCC cells. To further determine the function of FGF8 in human HCC cells, HepG2 and HCC-LM3 HCC cells were treated with various anticancer chemotherapeutic drugs, such as 5-FU, 

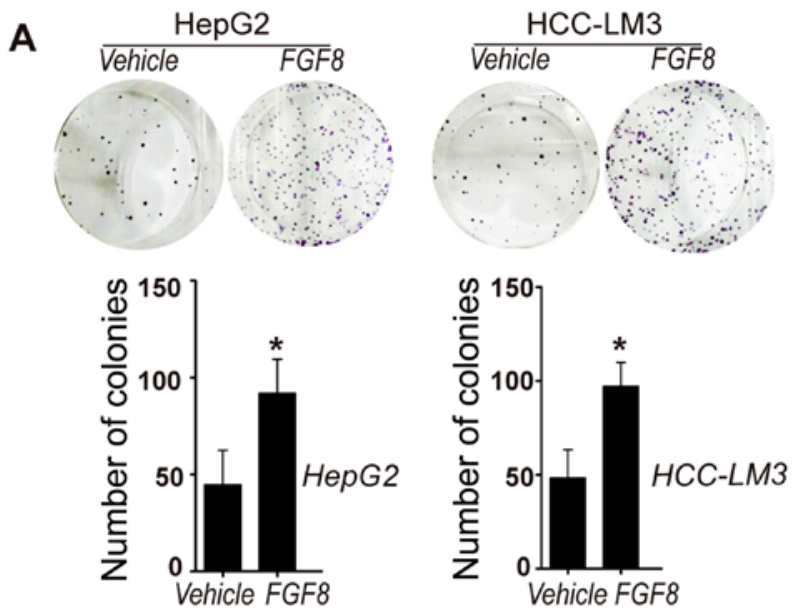

B
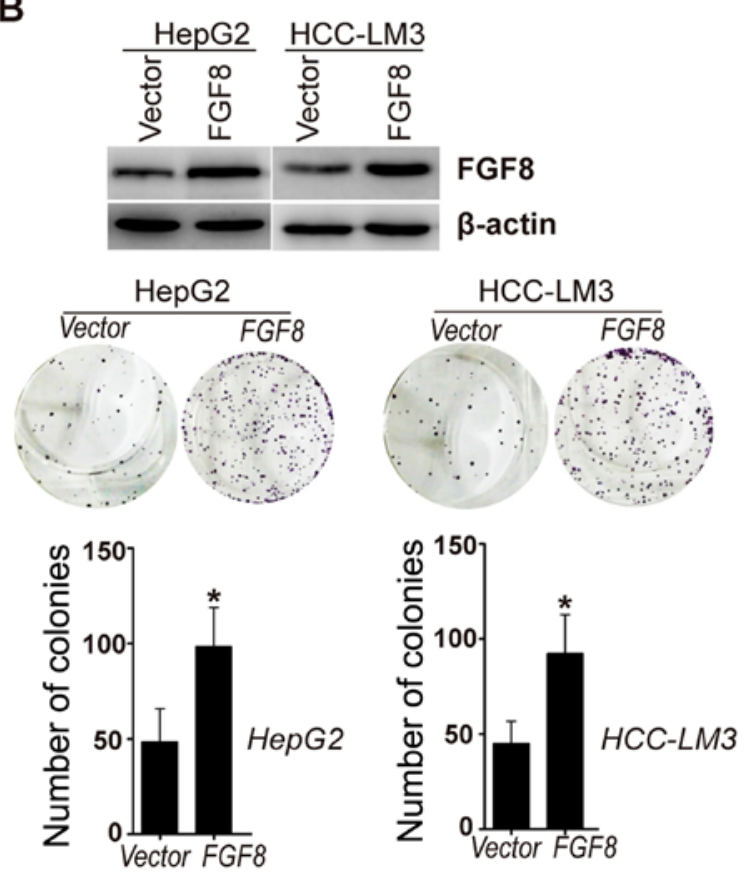

Figure 1. Effect of FGF8 on the proliferation of human HCC cells. (A) Exogenous recombinant FGF8 increased the proliferation of HepG2 and HCC-LM3 cells in vitro by colony formation assays. ${ }^{*} \mathrm{P}<0.01$. (B) The expression of FGF8 was determined by western blot analysis ( $\beta$-actin served as a loading control) in HepG2 or HCC-LM3 cells stably expressing FGF8. The proliferative activity was assessed by colony formation assay. $\mathrm{P}<0.01$. FGF8, fibroblast growth factor 8; HCC, hepatocellular carcinoma.

doxorubicin, paclitaxel, oxaliplatin, gefitinib (EGFR inhibitor) and RAD001 (Everolimus; mTOR inhibitor). CellTiter-Glo assays were used for the determination of cell viability. We found that exogenous recombinant FGF8 promoted the resistance to EGFR inhibitor gefitinib in HepG2 or HCC-LM3 HCC cells, but not to several other drugs (Fig. 2A). To confirm the effect of FGF8 on the resistance to EGFR inhibitors in human HCC cells, we performed colony formation assays to determine the effect of FGF8 in HepG2 or HCC-LM3 cells with another EGFR inhibitor erlotinib. As shown in Fig. 2B, erlotinib could not inhibit the proliferation of HepG2 cells with the treatment of exogenous recombinant FGF8.

FGF8 increases the expression of EGFR in human HCC cells. Gefitinib is a selective EGFR inhibitor and EGFR is found to be overexpressed in human HCC cells. Thus, we detected the expression levels of the EGFR protein with the treatment of exogenous recombinant FGF8 in HepG2 and HCC-LM3 HCC cells. The results revealed that the expression levels of the EGFR protein were increased by the stimulation of exogenous recombinant FGF8 in HepG2 and HCC-LM3 cells (Fig. 3A). In addition, HepG2 and HCC-LM3 cells stably overexpressing FGF8 also exhibited significantly increased expression levels of the EGFR protein compared with the vector control (Fig. 3B). Moreover, we determined the expression levels of EGFR mRNA by real-time PCR. Real-time PCR revealed significantly increased expression levels of EGFR mRNA in exogenous recombinant FGF8-treated HepG2 or HCC-LM3 cells (Fig. 3C; left panel). Consistently, the effect of stable overexpression of FGF8 was similar to the effect of exogenous recombinant FGF8 in HepG2 and HCC-LM3 cells (Fig. 3C; right panel).

FGF8 enhances the expression of EGFR via upregulation of YAP1. YAP1 is a transcriptional coactivator in the Hippo signaling pathway. Increased YAP1 expression can promote the growth of tumor cells. Moreover, YAP1 was demonstrated to mediate EGFR overexpression and confer chemoresistance in esophageal cancer cells (30). Thus, we detected the expression of YAP1 in HepG2 and HCC-LM3 cells with the treatment of exogenous recombinant FGF8. As shown in Fig. 4A, exogenous recombinant FGF8 upregulated the expression of the YAP1 protein. HepG2 and HCC-LM3 cells stably overexpressing FGF8 also exhibited upregulated expression of the YAP1 protein compared with the vector control (Fig. 4B). To further confirm the relationship between YAP1 and EGFR, endogenous expression of YAP1 was stably knocked down by lentivirus shRNAs in HepG2 or HCC-LM3 cells (Fig. 4C). We found that the expression of EGFR was inhibited in YAP1-knockdown cells (Fig. 4C). To determine whether FGF8-induced upregulation of EGFR was dependent upon its regulation to YAP1, HepG2 cells with stable knockdown of YAP1 were treated with exogenous recombinant FGF8. As shown in Fig. 4D, FGF8 could not regulate the expression of EGFR in HepG2 cells with stable knockdown of YAP1.

YAP1 is essential for FGF8 induced gefitinib resistance and $C S C$ properties. Upon determination that FGF8-induced upregulation of EGFR was dependent upon its regulation to YAP1, we then determined whether FGF8-induced gefitinib resistance in human HCC cells was dependent upon YAP1. CellTiter-Glo assays were used for the determination of cell viability after treatment with gefitinib. We found that exogenous recombinant FGF8 significantly increased the cell viability of gefitinib-treated vector control HepG2 and HCC-LM3 cells, but not YAP1-knockdown cells (Fig. 5A). Cancer stem cells (CSCs) are considered as the origin of cancer cells and one of the causes of multidrug resistance (31-34). The effects of EGFR on the maintenance of CSC properties has been demonstrated by other studies (35). Here, we investigated the effect of FGF8 on the CSC properties of HCC cells by tumor sphere formation assay. As shown in Fig. 5B, exogenous recombinant FGF8 significantly increased the tumor sphere formation of vector control HepG2 cells, but not YAP1-knockdown cells. 
A
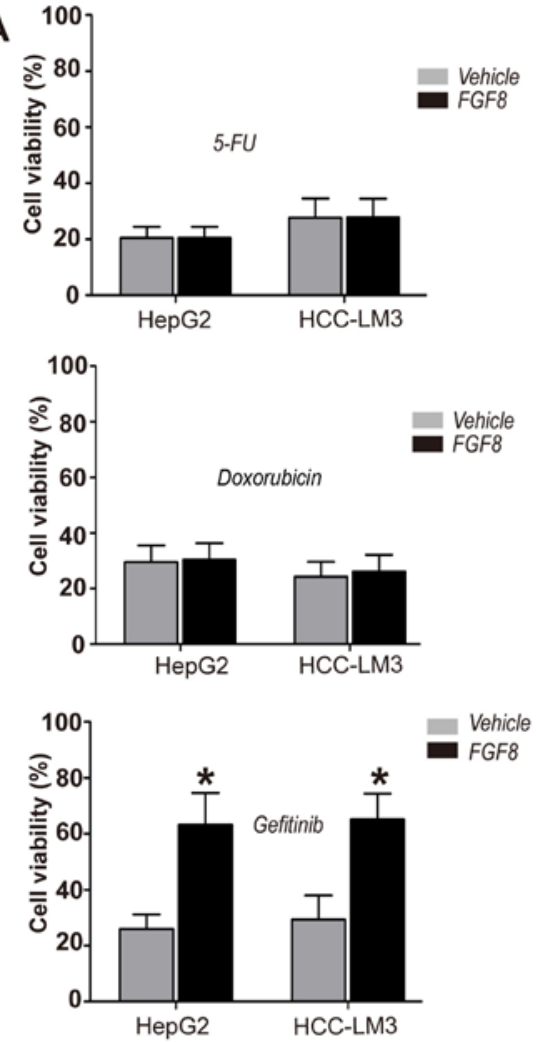

B

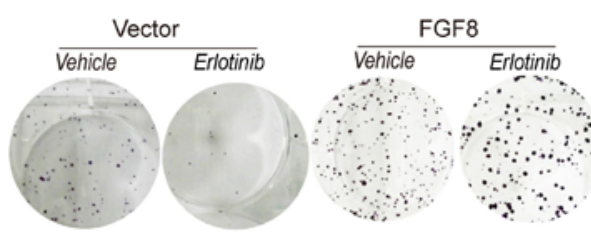

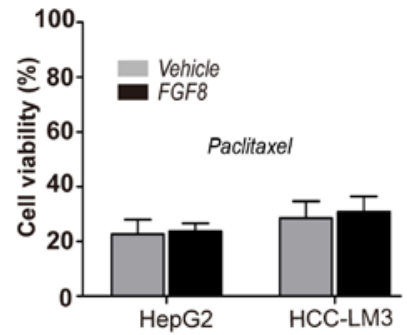
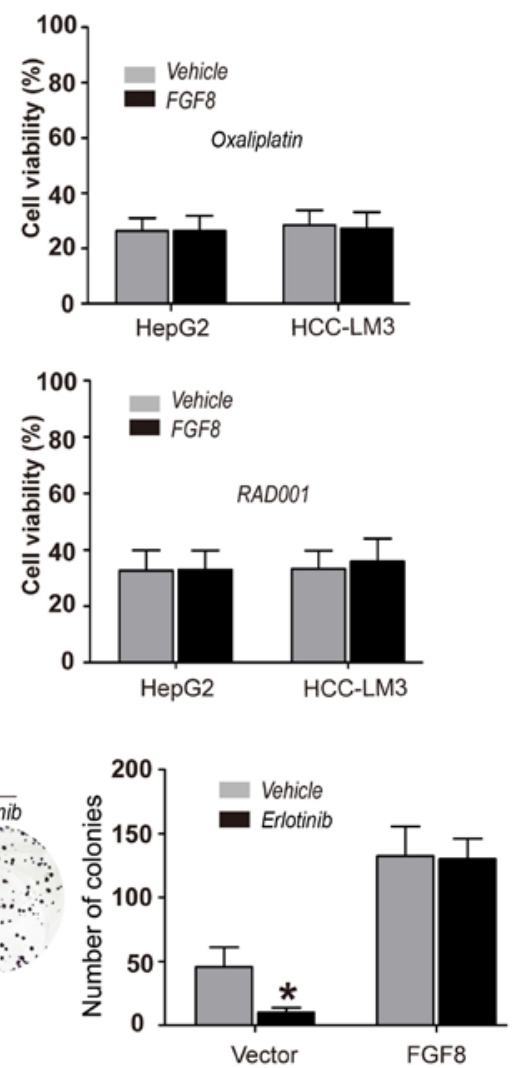

Figure 2. FGF8 promotes the resistance to EGFR inhibitors in human HCC cells. (A) CellTiter-Glo assays were used for the determination of the cell viability with the treatment of vehicle or exogenous recombinant human FGF8 $(250 \mathrm{ng} / \mathrm{ml})$ plus 5-fluorouracil $(5-\mathrm{FU}, 25 \mu \mathrm{g} / \mathrm{ml})$, doxorubicin $(20 \mu \mathrm{g} / \mathrm{ml})$, paclitaxel $(20 \mu \mathrm{g} / \mathrm{ml})$, oxaliplatin $(20 \mu \mathrm{g} / \mathrm{ml})$, gefitinib $(10 \mu \mathrm{g} / \mathrm{ml})$ or RAD001 (Everolimus; $100 \mu \mathrm{g} / \mathrm{ml})$. ${ }^{*} \mathrm{P}<0.02$. (B) Vector control or FGF8 stably expressing HepG2 or HCC-LM3 cells were treated with erlotinib $(10 \mu \mathrm{g} / \mathrm{ml})$ or the vehicle and the proliferative activity was assessed by colony formation assay. ${ }^{*} \mathrm{P}<0.005$. FGF8, fibroblast growth factor 8; HCC, hepatocellular carcinoma; EGFR, epidermal growth factor receptor.

A

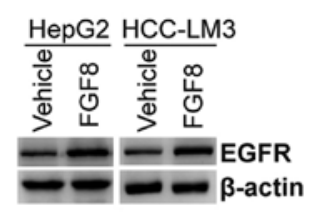

C

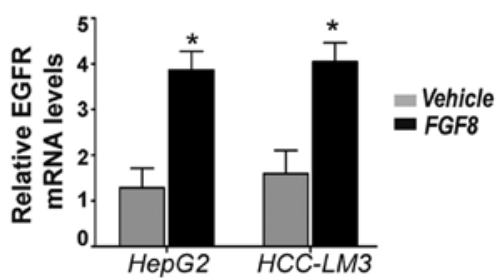

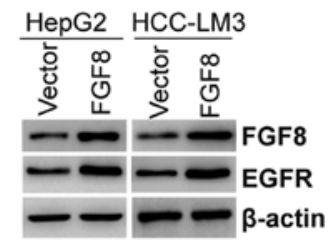

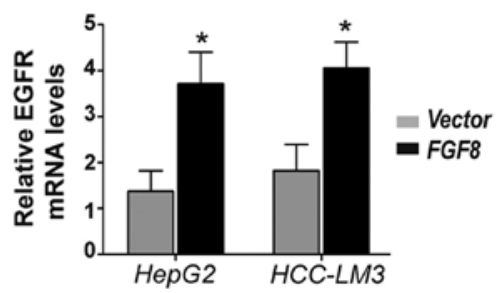

Figure 3. Effect of FGF8 on the expression of EGFR. (A) The expression of EGFR was determined by western blot analysis in vehicle or exogenous recombinant FGF8 treated $(250 \mathrm{ng} / \mathrm{ml})$ HepG2 or HCC-LM3 cells. $\beta$-actin served as a loading control. (B) The expression of FGF8 and EGFR was determined by western blot analysis in vector control, as well as in HepG2 and HCC-LM3 cells stably expressing FGF8. $\beta$-actin served as a loading control. (C) EGFR mRNA levels were determined by real-time PCR in exogenous recombinant FGF8-treated (250 ng/ml; left panel) and HepG2/HCC-LM3 cells stably expressing FGF8 (right panel). "P<0.01. FGF8, fibroblast growth factor 8; HCC, hepatocellular carcinoma; EGFR, epidermal growth factor receptor. 
A

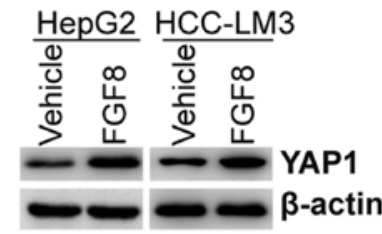

B

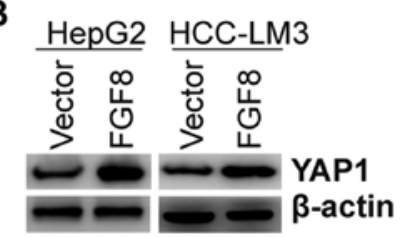

C

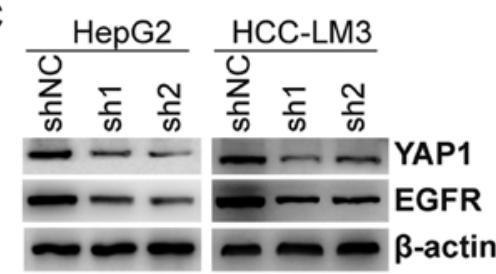

D

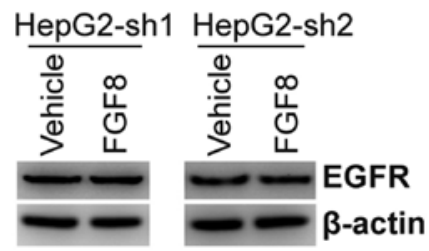

Figure 4. FGF8 enhances the expression of EGFR via upregulation of YAP1. (A) Expression of YAP1 levels were determined by western blot analysis in exogenous recombinant FGF8 $(250 \mathrm{ng} / \mathrm{ml})$ or the vehicle treated HepG2/HCC-LM3 cells. $\beta$-actin served as a loading control. (B) Expression of YAP1 levels were determined by western blot analysis in FGF8 stably expressing or the vector control HepG2/HCC-LM3 cells. $\beta$-actin served as a loading control. (C) Expression of YAP1 and EGFR levels were determined by western blot analysis in vector control (shNC) or different lentivirus-mediated YAP1-shRNA (sh1 and sh2) of HepG2/HCC-LM3 cells. $\beta$-actin served as a loading control. (D) Different lentivirus-mediated YAP1-shRNA (sh1 and sh2) of HepG2 cells were treated with recombinant FGF8 $(250 \mathrm{ng} / \mathrm{ml})$ or the vehicle. Expression of EGFR levels were determined by western blot anaysis. $\beta$-actin served as a loading control. FGF8, fibroblast growth factor 8; HCC, hepatocellular carcinoma; EGFR, epidermal growth factor receptor; YAP1, Yes-associated protein 1.

\section{Discussion}

HCC is one of the most commonly observed malignancies in the world, especially in developing countries $(36,37)$. Systemic treatments with chemotherapy (single agents or combination) have been used to treat advanced HCC, but these show no clear impact on overall survival $(38,39)$. Notably, HCCs are recognized as inherently chemotherapy-resistant (40). Additionally, CSCs are considered as the origin of cancer cells and one of the causes of multidrug resistance (39). Thus, some factors that determine CSC properties may play important roles in drug resistance to cancer chemotherapy.

FGF8 is a member of a large family of growth factors that signal through FGF tyrosine kinase receptors to regulate embryonic development, cell proliferation, cell differentiation and cell migration $(5,6,8)$. FGF8 is rarely detected in normal adult tissues, but is found overexpressed in several human tumors, including HCC. In the present study we first determined whether FGF8 could affect the proliferation of
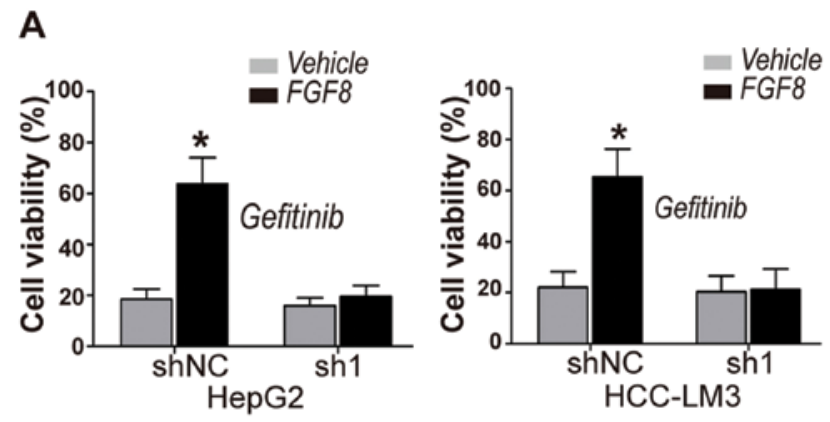

B

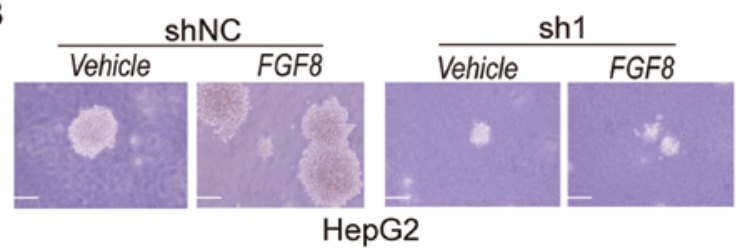

Figure 5. Essential function of YAP1 on FGF8-induced gefitinib resistance and CSC properties. (A) Vector control (shNC) or lentivirus-mediated shRNA to YAP1 (sh1) of HepG2/HCC-LM3 cells were treated with recombinant FGF8 $(250 \mathrm{ng} / \mathrm{ml})$ or the vehicle. CellTiter-Glo assays were used for the determination of cell viability. "P<0.02 (B) shNC or sh1-YAP1 of HepG2 cells were cultured under a non-adhesive culture system to form typical tumor spheres. Scale bar, $100 \mu \mathrm{m}$. YAP1, Yes-associated protein 1; FGF8, fibroblast growth factor 8; CSC, cancer stem cell; HCC, hepatocellular carcinoma.

human HCC cells. We found that stably overexpressing or exogenous recombinant FGF8 increased the proliferation of human HCC cells. 5-FU, doxorubicin, paclitaxel, oxaliplatin, gefitinib and RAD001 have been used in the treatment of cancer as anticancer chemotherapeutic drugs. We determined that exogenous recombinant FGF8 promoted the resistance to EGFR inhibitor gefitinib in HCC cells, but not to several other drugs. Additionally, the expression of EGFR was also found increased by exogenous recombinant or stably overexpressing FGF8.

YAP1 is a transcriptional coactivator in the Hippo signaling pathway (15). Increased YAP1 expression or activity can promote the growth of tumor cells, including human HCC $(14,16)$. In a previous study, FGF8 was found to promote colorectal cancer growth and metastasis by activating YAP1 signaling by increasing the transcription of YAP1 (6). Accordingly, expression of YAP1 was found to be increased in HCC cells by the stable overexpression or the exogenous addition of recombinant FGF8.

These findings imply that FGF8 enhances the resistance to EGFR inhibitors and the expression of EGFR via upregulation of YAP1. In the present study, stable knockdown of YAP1 by lentivirus shRNA inhibited FGF8-induced upregulation of EGFR and increased resistance to EGFR inhibitor gefinitib in HCC cells. In addition, we also found that FGF8 promoted the tumor sphere formation of HepG2 cells. Conversely, FGF8 could not increase the tumor sphere formation in YAP1knockdown cells.

In conclusion, we demonstrated that FGF8 promoted the proliferation of human HCC cells via upregulation of the YAP1/EGFR axis, thereby contributing to the resistance to EGFR inhibitors and CSC properties. Our results suggest an important role of FGF8 in the resistance to EGFR inhibition in human HCC cells. 


\section{Acknowledgements}

This study was funded by the Shanxi Health Department Research Project 201201003, and the Cooperation Project of Shandong University-Susceptible Gene of Breast Cancer of Chinese.

\section{References}

1. Sandhu DS, Baichoo E and Roberts LR: Fibroblast growth factor signaling in liver carcinogenesis. Hepatology 59: 1166-1173, 2014.

2. Yun YR, Won JE, Jeon E, Lee S, Kang W, Jo H, Jang JH, Shin US and Kim HW: Fibroblast growth factors: Biology, function, and application for tissue regeneration. J Tissue Eng 2010: 218142 , 2010.

3. Turner N and Grose R: Fibroblast growth factor signalling: From development to cancer. Nat Rev Cancer 10: 116-129, 2010.

4. Linscott ML and Chung WC: Fibroblast growth factor 8 expression in GT1-7 GnRH-secreting neurons is androgen-independent, but can be upregulated by the inhibition of DNA methyltransferases. Front Cell Dev Biol 4: 34, 2016.

5. Chen N, Ma J, Zhao Y, Wu M, Yang H, Gong W, Chao J and Li X: Expression of functional recombinant human fibroblast growth factor $8 \mathrm{~b}$ and its protective effects on $\mathrm{MPP}^{+}$-lesioned $\mathrm{PC} 12$ cells. Appl Microbiol Biotechnol 100: 625-635, 2016.

6. Liu R, Huang S, Lei Y, Zhang T, Wang K, Liu B, Nice EC, Xiang $\mathrm{R}, \mathrm{Xie} \mathrm{K}$, Li J, et al: FGF8 promotes colorectal cancer growth and metastasis by activating YAP1. Oncotarget 6: 935-952, 2015.

7. Zhong C, Saribekyan G, Liao CP, Cohen MB and Roy-Burman P: Cooperation between FGF8b overexpression and PTEN deficiency in prostate tumorigenesis. Cancer Res 66: 2188-2194, 2006.

8. Dorkin TJ, Robinson MC, Marsh C, Bjartell A, Neal DE and Leung HY: FGF8 over-expression in prostate cancer is associated with decreased patient survival and persists in androgen independent disease. Oncogene 18: 2755-2761, 1999.

9. Mo JS, Park HW and Guan KL: The Hippo signaling pathway in stem cell biology and cancer. EMBO Rep 15: 642-656, 2014.

10. Zhao B, Li L, Lei Q and Guan KL: The Hippo-YAP pathway in organ size control and tumorigenesis: An updated version. Genes Dev 24: 862-874, 2010.

11. Zhang H, Pasolli HA and Fuchs E: Yes-associated protein (YAP) transcriptional coactivator functions in balancing growth and differentiation in skin. Proc Natl Acad Sci USA 108: 2270-2275, 2011.

12. Nguyen Q, Anders RA, Alpini G and Bai H: Yes-associated protein in the liver: Regulation of hepatic development, repair, cell fate determination and tumorigenesis. Dig Liver Dis 47: 826-835, 2015.

13. Kowalik MA, Saliba C,Pibiri M,Perra A,Ledda-Columbano GM, Sarotto I, Ghiso E, Giordano S and Columbano A: Yes-associated protein regulation of adaptive liver enlargement and HCC development in mice. Hepatology 53: 2086-2096, 2011.

14. Rosenbluh J, Nijhawan D, Cox AG, Li X, Neal JT, Schafer EJ, Zack TI, Wang X, Tsherniak A, Schinzel AC, et al: $\beta$-catenin-driven cancers require a YAP1 transcriptional complex for survival and tumorigenesis. Cell 151: 1457-1473, 2012.

15. Kapoor A, Yao W, Ying H, Hua S, Liewen A, Wang Q, Zhong Y, Wu CJ, Sadanandam A, Hu B, et al: Yap1 activation enables bypass of oncogenic Kras addiction in pancreatic cancer. Cell 158: 185-197, 2014

16. Lorenzetto E, Brenca M, Boeri M, Verri C, Piccinin E, Gasparini P, Facchinetti F, Rossi S, Salvatore G, Massimino M, et al: YAP1 acts as oncogenic target of 11q22 amplification in multiple cancer subtypes. Oncotarget 5: 2608-2621, 2014.

17. Panvichian R, Tantiwetrueangdet A, Sornmayura P and Leelaudomlipi S: Missense mutations in exons 18-24 of EGFR in HCC tissues. Biomed Res Int 2015: 171845, 2015.

18. Lanaya H, Natarajan A, Komposch K, Li L, Amberg N, Chen L, Wculek SK, Hammer M, Zenz R, Peck-Radosavljevic M, et al: EGFR has a tumour-promoting role in liver macrophages during HCC formation. Nat Cell Biol 16: 972-981, 2014.
19. Badawy AA, El-Hindawi A, Hammam O, Moussa M, Gabal S and Said N: Impact of epidermal growth factor receptor and transforming growth factor- $\alpha$ on hepatitis $C$ virus-induced hepatocarcinogenesis. APMIS 123: 823-831, 2015.

20. Thomas MB, Morris JS, Chadha R, Iwasaki M, Kaur H, Lin E, Kaseb A, Glover K, Davila M and Abbruzzese J: Phase II trial of the combination of bevacizumab and erlotinib in patients who have advanced HCC. J Clin Oncol 27: 843-850, 2009.

21. Yau T, Wong H, Chan P, Yao TJ, Pang R, Cheung TT, Fan ST and Poon RT: Phase II study of bevacizumab and erlotinib in the treatment of advanced HCC patients with sorafenib-refractory disease. Invest New Drugs 30: 2384-2390, 2012.

22. Zhang J, Zong Y, Xu GZ and Xing K: Erlotinib for advanced HCC. A systematic review of phase II/III clinical trials. Saudi Med J 37: 1184-1190, 2016.

23. Llovet JM and Bruix J: Testing molecular therapies in HCC: The need for randomized phase II trials. J Clin Oncol 27: 833-835, 2009.

24. Gu HR, Park SC, Choi SJ, Lee JC, Kim YC, Han CJ, Kim J, Yang KY, Kim YJ, Noh GY, et al: Combined treatment with silibinin and either sorafenib or gefitinib enhances their growth-inhibiting effects in HCC cells. Clin Mol Hepatol 21: 49-59, 2015.

25. Wei Z, Doria C and Liu Y: Targeted therapies in the treatment of advanced HCC. Clin Med Insights Oncol 7: 87-102, 2013.

26. Ezzoukhry Z, Louandre C, Trécherel E, Godin C, Chauffert B, Dupont S, Diouf M, Barbare JC, Mazière JC and Galmiche A: EGFR activation is a potential determinant of primary resistance of HCC cells to sorafenib. Int J Cancer 131: 2961-2969, 2012.

27. Yu HC, Chen HJ, Chang YL, Liu CY, Shiau CW, Cheng AL and Chen KF: Inhibition of CIP2A determines erlotinib-induced apoptosis in HCC. Biochem Pharmacol 85: 356-366, 2013.

28. Huether A, Höpfner M, Sutter AP, Schuppan D and Scherübl H: Erlotinib induces cell cycle arrest and apoptosis in hepatocellular cancer cells and enhances chemosensitivity towards cytostatics. J Hepatol 43: 661-669, 2005.

29. Zhao J, Kelnar K and Bader AG: In-depth analysis shows synergy between erlotinib and miR-34a. PLoS One 9: e89105, 2014.

30. Song S, Honjo S, Jin J, Chang SS, Scott AW, Chen Q, Kalhor N, Correa AM, Hofstetter WL, Albarracin CT, et al: The Hippo coactivator YAP1 mediates EGFR overexpression and confers chemoresistance in esophageal cancer. Clin Cancer Res 21: 2580-2590, 2015

31. Liu X, Feng D, Liu D, Wang S, Yu X, Dai E, Wang J, Wang L and Jiang W: Dissecting the origin of breast cancer subtype stem cell and the potential mechanism of malignant transformation. PLoS One 11: e0165001, 2016.

32. Song K, Wu J and Jiang C: Dysregulation of signaling pathways and putative biomarkers in liver cancer stem cells (Review). Oncol Rep 29: 3-12, 2013

33. Szakács G, Paterson JK, Ludwig JA, Booth-Genthe C and Gottesman MM: Targeting multidrug resistance in cancer. Nat Rev Drug Discov 5: 219-234, 2006.

34. Di $\mathrm{C}$ and Zhao Y: Multiple drug resistance due to resistance to stem cells and stem cell treatment progress in cancer (Review). Exp Ther Med 9: 289-293, 2015.

35. Ma L, Zhang G, Miao XB, Deng XB, Wu Y, Liu Y, Jin ZR, Li XQ, Liu QZ, Sun DX, et al: Cancer stem-like cell properties are regulated by EGFR/AKT/ $\beta$-catenin signaling and preferentially inhibited by gefitinib in nasopharyngeal carcinoma. FEBS J 280: 2027-2041, 2013.

36. Zhu RX, Seto WK, Lai CL and Yuen MF: Epidemiology of HCC in the Asia-Pacific Region. Gut Liver 10: 332-339, 2016.

37. Gong XL and Qin SK: Progress in systemic therapy of advanced HCC. World J Gastroenterol 22: 6582-6594, 2016.

38. Deng GL, Zeng S and Shen H: Chemotherapy and target therapy for HCC: New advances and challenges. World J Hepatol 7: 787-798, 2015.

39. Brown KS: Chemotherapy and other systemic therapies for HCC and liver metastases. Semin Intervent Radiol 23: 99-108, 2006.

40. Pardee AD and Butterfield LH: Immunotherapy of HCC: Unique challenges and clinical opportunities. Oncoimmunology 1 : 48-55, 2012. 